\title{
Diseño e implementación de un sistema de gestión de calidad académico administrativa en la Unidad de Posgrado de la Facultad de Ingeniería Industrial de la UNMSM
}

\section{RESUMEN}

La Unidad de Posgrado de la Facultad de Ingeniería Industrial de la UNMSM (UPG) a partir del año 2007 experimentó un crecimiento acelerado en cuanto a alumnos y programas, en este escenario, la gestión académica y administrativa que se venía llevando resultó insuficiente e inadecuada a los enfoques modernos de la educación superior; esto propició la siguiente pregunta ¿Qué diseño de gestión es adecuado y como debe implementarse en la UPG tomando como base la norma ISO 9001?. Para ello se aplicaron los conceptos de mejoramiento continuo y análisis del mapa de procesos, obteniéndose como resultado la validación del Sistema de Gestión de Calidad Académica y Administrativa en dos semestres académicos consecutivos

Palabras claves: Calidad educativa, norma de calidad, proceso de gestión académica, gestión organizacional.

DESIGN AND IMPLEMENTATION OF A MANAGEMENT SYSTEM OF ACADEMIC QUALITY MANAGEMENT IN THE UNITED GRADUATE FACULTY OF ENGINEERING OF THE UNMSM

\section{ABSTRACT}

The industrial faculty posgrade unit of UNMSM (UPG) since 2007 had an accelerated growing in programs and students, in this scenario, the academic - management system was not enough to the higher education modern approach, generating the next question: What kind of quality management design is appropriate and how to must be implemented at the UPG taking as a base the ISO 9001 standard?. In order to answer this question, the work team applied concepts like continuously improvement and the process map analysis ; having as a result the validation of the System Management of Academic - Administrative Quality in two academic semesters.

Keywords: Norm, quality, document, process, management.

\section{INTRODUCCIÓN}

El estudio del diseño y la implementación de un Sistema de Gestión basado en la norma ISO 9001 o Sistema de Gestión de Calidad (SGC) es un tema relevante porque constituye la estructura fundamental para brindar un buen servicio en la UPG. El objetivo del presente artículo es mostrar en forma breve los procesos seguidos, tanto para el diseño como para la implementación, incluyendo algunos criterios fundamentales y los resultados luego de la fase de validación, realizado en dos semestres académicos consecutivos.

EI SGC descrito en el presente artículo puede servir como modelo para otras Unidades de Posgrado con problemas similares a los experimentados por la UPG, pero sobre todo puede servir como una guía para el análisis y la formulación de sistemas similares.

La Facultad de Ingeniería Industrial de la UNMSM, desde el año 1987 y a través de la UPG viene desarrollando programas de posgrado. Hasta el año 2007 estos programas se restringían a dos: Maestría en Ingeniería Industrial (con menciones en Producción y Gestión Industrial) y Doctorado en Ingeniería Industrial; sin embargo, y debido a la creciente demanda de profesionales capacitados en diferentes áreas relacionadas a la ingeniería industrial, se vio la necesidad de aumentar la oferta de programas ofrecidos por la UPG, lo cual trajo un aumento en la cantidad de estudiantes y en cambios estructurales en el sistema de gestión que se venía utilizando.

\section{POLÍTICA DE CALIDAD DE LA UPG}

A partir del diagnóstico efectuado previamente, de las recomendaciones e ideas tanto del personal docente como no docente, estudiantes y egresados de la UPG recogidas en reuniones de coordinación y además considerando el Plan Estratégico, tanto de la Facultad como de la UNMSM, se formuló la siguiente política de calidad:

"La Unidad de Posgrado se compromete a satisfacer las expectativas de sus alumnos a través de una formación académica de

1 Doctor en Ciencias Administrativas. Profesor del Departamento de Diseño y Tecnología Industrial - UNMSM. E-mail: jlinche@hotmail.com

2 Magíster en Ingeniería Industrial. Profesor del Departamento de Diseño y Tecnología Industrial. UNMSM. E-mail: ramon_chung@yahoo.es

3 Ingeniero Industrial. Profesor del Departamento de Producción y Gestión Industrial. UNMSM. E-mail: jasalasb@hotmail.com 
excelencia acorde con la misión de la UNMSM, en cumplimiento con los requisitos legales y reglamentarios, contando para ello con el apoyo de docentes competentes y con un Sistema de Gestión de la Calidad en mejora continua".

La política de calidad es de gran importancia, puesto que orientará el mapa de procesos, el cual será la matriz de todo el sistema a diseñarse. Asimismo, está dirigida a la satisfacción del cliente, lo cual se logra mediante docentes competentes, constantemente capacitados y evaluados y con un SGC dentro de la filosofía de la mejora continua.

\section{MAPA DE PROCESOS}

El mapa de procesos constituye la estructura fundamental que permite el cumplimiento de la políti- ca de calidad formulada anteriormente; para esto fue necesario identificar los procesos globales en el nuevo sistema a diseñar y cómo se relacionan entre ellos en el marco de dicha política.

Dentro del análisis se identificaron los siguientes procesos:

1. Diseño y desarrollo

2. Planificación

3. Admisión

4. Enseñanza

5. Evaluación del programa

La entrada del mapa está dada por los requerimientos del cliente (el cual está representado por el alumno de la UPG) y la salida por clientes satisfechos. Para un mejor entendimiento, véase la Figura 1.

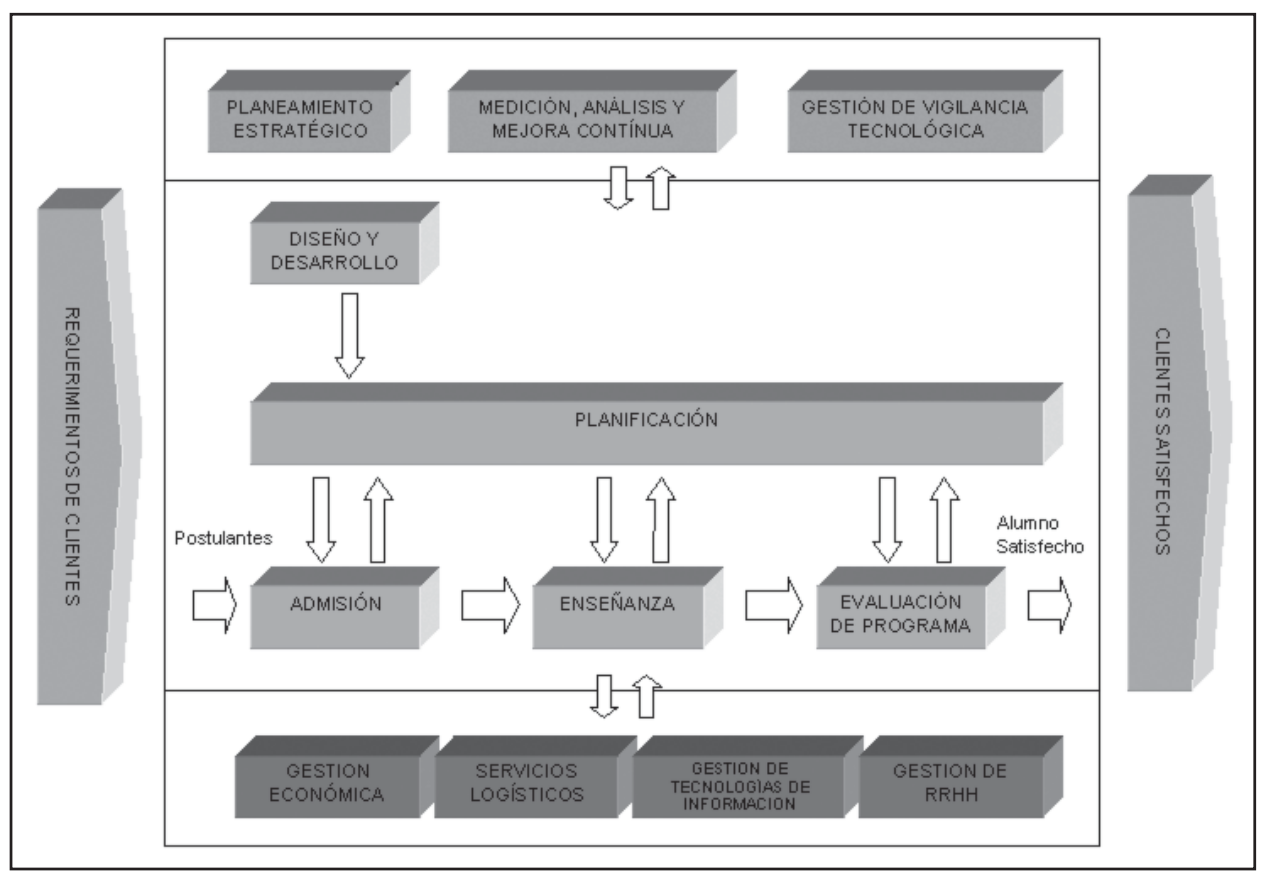

Fuente: Elaboración propia.

Figura 1. Mapa de procesos de la UPG.

Como se puede apreciar en la Figura 1, el mapa de procesos involucra las unidades orgánicas: gestión económica (Oficina de Economía), servicios logísticos (Oficina de Servicios Generales y Mantenimiento), gestión de tecnologías de información (Oficina de Tecnología Educativa) y gestión de recursos humanos (Oficina de Personal); asimismo recibe información de la Oficina de Planificación.

\section{PROCEDIMIENTOS DE CALIDAD}

Los procedimientos de calidad son los instrumentos que en la práctica permiten la ejecución del mapa de procesos de la UPG; como se mencionó anteriormente, por cada proceso de calidad se ha elaborado un procedimiento para su realización; asimismo, son complementados por otros procedimientos de carácter secundario. 
1. Procedimientos principales

- Admisión para los programas de Maestría y de Doctorados

- Diseño y desarrollo de programas de Maestría

- Planificación del programa

- Enseñanza

- Evaluación del programa

- Procedimiento para la obtención del grado académico de Magíster y Doctor.

\section{Procedimientos secundarios}

\section{- Control de Archivos}

- Control de Documentos

Para el funcionamiento de los procedimientos, así como para la estandarización de reportes e informes, el SGC utiliza formatos que facilitan el trabajo de recolección de datos y la presentación de informes.

En la elaboración de los procedimientos se han considerado documentos externos a la UPG, tales como: El Estatuto de la UNMSM, el Reglamento General de Estudios de Posgrado aprobado con RR 00301-R-09, el Plan Estratégico de la Facultad de Ingeniería Industrial (2007 - 2011), entre otros.

EI SGC cuenta con un módulo en Internet dentro del campus virtual de la UPG (http://campusvirtualfii.unmsm.edu.pe/virtualupg/) en el cual se pueden descargar los procedimientos, formatos, normas entre otros representando un soporte muy importante para la ejecución de los diversos procedimientos y para otras consultas adicionales. Si bien todos estos documentos se encuentran archivados físicamente, la versión digital permite consultas remotas.

\section{LA GESTIÓN DOCUMENTARIA EN EL SGC}

La gestión documentaria en el SGC de la UPG ordena la información y establece los procedimientos para su uso, distribución y almacenamiento. Las normas internas que rigen este procedimiento son:

PR-SGC-02-UPG-FII-08: Control de archivos

\section{PR-SGC-01-UPG-FII-08: Control de documentos}

La primera norma se encarga de regular el sistema General de Archivos (SGA), el cual define al Documento como medio que contiene información y al Archivo como la colección de documentos agrupados según una característica común.
EI SGA de la UPG contiene los siguientes archivos:

a) Programa por año.

b) Expediente del alumno.

c) Archivo de encuestas.

d) Procedimiento para Gestión de Calidad.

e) Expediente del docente.

f) Normas de la UPG

g) Archivo de la jefatura académica.

También establece el contenido y orden de cada uno de ellos así como su etiquetado, preparación, tiempo de vida y disposición final.

La segunda norma indica el procedimiento para la elaboración de documentos, su contenido, encabezados, pie de página entre otros; además establece la clave que se le debe asignar.

\section{LA PIRÁMIDE DOCUMENTARIA INTERNA}

La jerarquía documentaria en un SGC es importante para poder comprender mejor el sistema, en el caso de la UPG, la Figura 2 muestra gráficamente dicha pirámide.

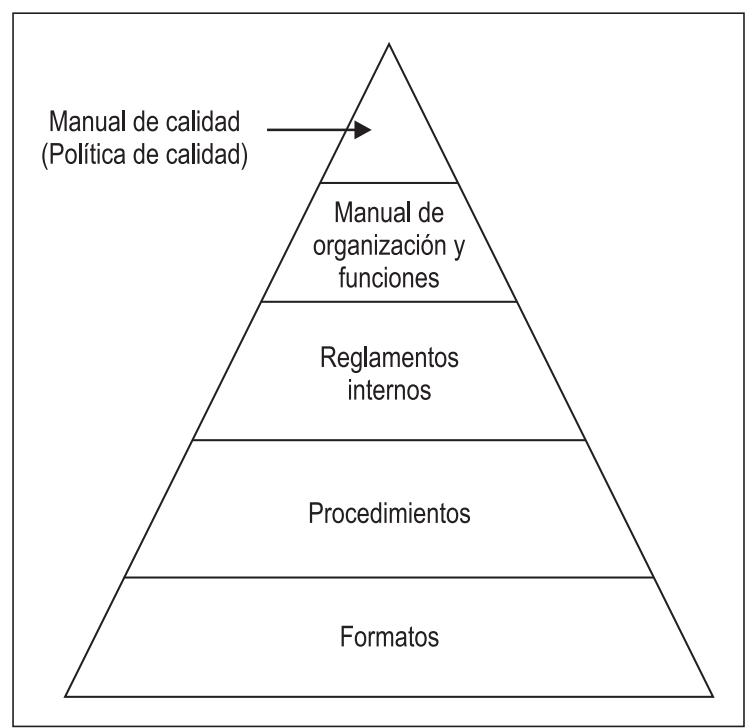

Fuente: Elaboración propia basada en la norma ISO 9001:2007

Figura 2. Pirámide documentaria del SGC-UPG.

En la Figura 2 se puede apreciar que el Manual de Calidad sirve como referencia de la orientación del SGC, el Manual de Organización y Funciones 
(MOF) es el instrumento normativo que permite la realización de la política de calidad.

Asimismo, los reglamentos internos fueron elaborados tomando en cuenta el MOF, entre otros documentos propios de la Facultad y de la UNMSM.
Con toda esta información (Plan estratégico, mapa estratégico, MOF y reglamentos) se detallan los procedimientos y los formatos; El Cuadro 1 presenta en forma resumida el sistema documentario.

Cuadro 1. Esquema del sistema documentario.

\begin{tabular}{|c|c|c|c|}
\hline PROCESO & ARCHIVO & CONTENIDO & FORMATO \\
\hline $\begin{array}{l}\text { - Planificación } \\
\text { - Admisión } \\
\text { - Enseñanza } \\
\text { - Evaluación }\end{array}$ & Programa por año & $\begin{array}{l}\text { - Sílabo del curso } \\
\text { - Acta Interna de notas } \\
\text { - Informe final de encuestas } \\
\text { - Listado de asistencia } \\
\text { - Informe del docente } \\
\text { - Informe de uso del Campus Virtual }\end{array}$ & $\begin{array}{l}\text { - RE-OPE-01-UPG-FII-09 } \\
\text { - RE-OPE-03-UPG-FII-09: Acta interna de notas } \\
\text { - IN-OPE-01-UPG-FII-09: Resultados de encuesta } \\
\text { - RE-OPE-02-UPG-FII-09: Formato de asistencia } \\
\text { - IN-OPE-03-UPG-FII-09: Informe del docente } \\
\text { - IN-OPE-02-UPG-FII-09: informe del uso campus virtual }\end{array}$ \\
\hline $\begin{array}{l}\text { - Planificación } \\
\text { - Admisión }\end{array}$ & Expediente del alumno & $\begin{array}{l}\text { - Lista de verificación del expediente del } \\
\text { postulante. } \\
\text { - Ficha 1: datos del postulante y declaración } \\
\text { jurada. } \\
\text { - Copia del título profesional fedateada por } \\
\text { la universidad de origen. } \\
\text { - Certificado de estudio fedateado por la } \\
\text { universidad de origen. } \\
\text { - Copia notariada del D.N.I. } \\
\text { - Currículo vitae documentado. } \\
\text { - Compromiso de Honor firmado. } \\
\text { - Otros documentos relacionados al alumno. }\end{array}$ & $\begin{array}{l}\text { En todos los casos se utiliza el formato proporcionado } \\
\text { por la Escuela de Posgrado de la UNMSM }\end{array}$ \\
\hline Evaluación & Archivo de Encuestas & Encuestas & RE-OPE-05-UPG-FII-09: Encuestas \\
\hline Todos los procesos & $\begin{array}{l}\text { Procedimientos de } \\
\text { Gestión de Calidad }\end{array}$ & $\begin{array}{l}\text { Este archivo se divide en dos partes: } \\
\text { - Procedimientos } \\
\text { - Formatos }\end{array}$ & No aplica \\
\hline Enseñanza & Expediente del Docente & Hoja de vida del docente & RE-OPE-04-UPG-FII-09: Hoja de vida del docente \\
\hline Todos los procesos & Normas de la UPG & Reglamentos y manuales & Formatos propios de los reglamentos y manuales \\
\hline $\begin{array}{l}\text { - Diseño y Desarrollo } \\
\text { - Evaluación }\end{array}$ & $\begin{array}{l}\text { Archivo de la jefatura } \\
\text { académica }\end{array}$ & $\begin{array}{l}\text { El archivo registra informes diversos de } \\
\text { Diseño y Desarrollo así como los informes } \\
\text { de evaluación del ciclo académico, informe } \\
\text { del programa entre otros. }\end{array}$ & Formatos varios \\
\hline
\end{tabular}

Fuente: Elaboración propia.

\section{VALIDACIÓN DEL SGC}

La validación del SGC se llevó a cabo durante dos ciclos académicos; en dicho proceso se pudieron observar dos puntos fundamentales:

a) Los procedimientos, así como varios documentos internos tuvieron que modificarse para adecuarse mejor a la realidad. b) El personal administrativo tuvo una fase inicial de asimilación e involucramiento en el nuevo sistema de trabajo.

Para la validación se utilizaron diversas listas de chequeo; el cuadro 2 presenta una de dichas listas aplicada al archivo de programa por año de la maestría en Ingeniería Industrial 
Cuadro 2. Lista de chequeo para la maestría en Ingeniería Industrial.

\begin{tabular}{|c|c|c|c|c|c|c|}
\hline Curso & Docente / Documento & 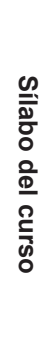 & 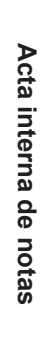 & 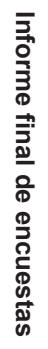 & 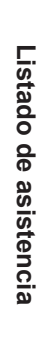 & 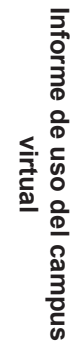 \\
\hline Gerencia de marketing & Roger Arévalo Ramírez & $x$ & $\mathrm{x}$ & $x$ & $\mathrm{x}$ & $\mathrm{x}$ \\
\hline Gestión tecnológica & Aristides Sotomayor & $\mathrm{x}$ & $\mathrm{x}$ & $\mathrm{x}$ & $\mathrm{x}$ & $\mathrm{x}$ \\
\hline Costo para la toma de decisiones & Javier del Carpio & $\mathrm{x}$ & $\mathrm{x}$ & $\mathrm{x}$ & $\mathrm{x}$ & $\mathrm{x}$ \\
\hline Innovación de productos de procesos & Carlos Gálvez & $\mathrm{x}$ & $\mathrm{x}$ & $\mathrm{x}$ & $\mathrm{x}$ & $\mathrm{x}$ \\
\hline Taller de tesis II & Francisca Bouby & $x$ & $x$ & $x$ & $x$ & $x$ \\
\hline Seguridad y mantenimiento industrial & Christian Navarro & $x$ & $x$ & $x$ & $x$ & $x$ \\
\hline Logística & José Falcón & $x$ & $x$ & $x$ & $x$ & $x$ \\
\hline Psicología industrial & Gissela Medina Soto & $x$ & $x$ & $x$ & $x$ & $x$ \\
\hline Taller de tesis II & Juan Cevallos Ampuero & $x$ & $x$ & $x$ & $x$ & $x$ \\
\hline Análisis y diseño de sistemas emp. & Hilmar Hinojosa Lazo & $x$ & $x$ & $x$ & $x$ & $\mathrm{x}$ \\
\hline Modelos de optimización & Oscar Gamonal & $x$ & $x$ & $x$ & $x$ & $x$ \\
\hline Gestión de la calidad & Alejandro Gallegos & $\mathrm{x}$ & $\mathrm{x}$ & $\mathrm{x}$ & $\mathrm{x}$ & $\mathrm{x}$ \\
\hline Planeamiento de la investigación & Teonila García & $\mathrm{x}$ & $\mathrm{x}$ & $\mathrm{x}$ & $\mathrm{x}$ & $\mathrm{x}$ \\
\hline
\end{tabular}

Al finalizar el periodo de prueba, el SGC quedó validado y listo para su puesta en marcha durante el siguiente ciclo, sin embargo es necesario aclarar que no es un sistema cerrado, muy por el contrario, es un sistema que debe adaptarse a los cambios de un escenario dinámico, lo cual implicará un proceso permanente de mejora continua.

EI SGC abarca sólo los principales procesos de los programas de maestrías, por lo tanto, el siguiente paso es extender el sistema al resto de procesos y a los programas de doctorado.

\section{CONCLUSIONES Y RECOMENDACIONES}

1. El diseño de un SGC debe ser contrastado con la realidad a fin de ajustarlo y dejarlo listo para su funcionamiento.

2. El cambio que involucra un Sistema de Gestión "tradicional" a un SGC involucra no solo cambios en los procedimientos, sino también en la forma de hacer las cosas y en las actitudes del personal involucrado; es decir cambios en los individuos que conforman la organización.

3. El personal es el elemento más importante en un SGC por lo tanto su capacitación y sensibilización son de suma importancia.

4. Un SGC no es un sistema estático, sino dinámico y regido por el principio de la mejora continua.

5. Es recomendable que un SGC tome como base los procedimientos del Sistema de Gestión que se piensa reemplazar, a fin de analizarlos y adaptarlo o modificarlo a la nueva filosofía que el cambio involucra.

6. Todo SGC desde su fase inicial debe ser diseñado de tal forma que no entre en conflicto con normas de carácter superior (estatutos, normas generales, plan estratégico etc.), sino por el contrario debe tener la misma orientación y desarrollarse dentro de ese marco. 


\section{REFERENCIAS BIBLIOGRÁFICAS}

[1] AENOR (2008). UNE - EN ISO 9001:2008. AENOR. España

[2] UNMSM (1984). Estatuto de la UNMSM. Diario Oficial El Peruano (septiembre 1984). Lima, Perú.

[3] UNMSM - EPG (2009). Reglamento General de Estudios de Posgrado.
[4] Cáceres J. (2008). Gestión de la Calidad. Red Científica Peruana. En: www.rcp.net.pe/downloads/Gestion_dc.ppt (visitado el 13-02-2009).

[5] Caballano J. (2008). Gestión de la Calidad. En: www.elprisma.com/apuntes/administracion_de empresas/gestiondelacalidad. (Visitado el 2203-2009)

[6] ISO (2007). Norma ISO 9001:2007. INDECOPI. Lima, Perú 